from a particular solution* of his equation for $\psi$ and corresponds to the case of rectilinear motions of the particles of the swarm as viewed by an observer of class $A$. This is therefore the case when the interactions of the particles are neglected. It follows that at $t=0$ there were an infinite number of particles at the origin. At any subsequent $t>0$, particles which were initially at the origin and moving with velocity $V$, will have got to $r=V t$ and evidently these particles again have an infinity in their distribution at that distance. Thus these infinities recede from the observer with velocities proportional to their distances from him. The general effect of gravitation is to remove these infinities and to replace them by density-maxima in the distribution of the particles. Thus finally we have a set of groups of the original particles which appear to any observer of class $A$ to recede from him according to the law $r=V t$. However, there still remain singularities in the distribution of the groups on the sphere $r=c t$, so that the latter are strongly concentrated there. Milne identifies the groups with the spiral nebulæ, thus obtaining the law of velocity of recession proportional to distance. The concentration towards $r=c t$ is shown to be consistent with the apparent uniform distribution of the nebulæ owing to the rapid diminution of

* It appears as the only solution in the paper quoted in (2). Prof Milne has since discovered others. brightness due to the high velocity an object acquires on approaching this bounding sphere.

From the observed correlation of $500 \mathrm{~km}$. per sec. for every 3.25 million light years distance of a nebula and the law $r=V t$, it follows that $t=2 \times 10^{9}$ years have elapsed since the system of the nebulæ was at its greatest concentration.

We may in conclusion contrast Milne's theory with that based on general relativity. The latter deals only with the group of observers at rest with respect to one another, who are observing a set of particles also at rest with respect to the observers in the sense that the total momentum of the particles is assumed to be zero. The particles initially are in a state of unstable equilibrium and the expansion is due to what may be called a 'cosmical repulsion'. In Milne's theory the particles are initially in motion and are not in an equilibrium state. The observers considered are not at rest with respect to each other. The expansion is simply due to the fact that the particles in a certain region have higher velocities on the whole than those elsewhere. Both theories are in accordance with observation and it seems impossible to decide definitely for or against either so long as the phenomenon of the recession of the nebulæ, in isolation from all other phenomena, is to be the only criterion.

${ }^{1}$ NATURE, 130, 2-10, July 2, and 507-508, Oct. 1, 1932.

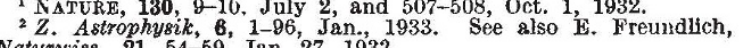
Naturwiss., 21, 54-59, Jan. 27, 1933.

\title{
Nitrogen-Uptake of Plants
}

$\mathrm{P}$ ROGRESS, due to the abandonment of traditional beliefs, is occurring to-day in agrieulture as well as in other branches of applied science. At one time it was generally held that ammoniacal nitrogen had first to be converted into nitric nitrogen before it could be taken up by plants, but we now know of many plants that take up ammoniacal nitrogen directly; and at the present time doubt is being cast on a doctrine which has persisted since the days of Liebig and Boussingault, namely, that organic nitrogen has first to be 'mineralised' before it becomes available as food for plants. The work done by Prof. A. I. Virtanen and his collaborators at Helsingfors during the past few years has provided important evidence that at least some plants directly and readily assimilate organic nitrogenous compounds, and a useful summary of this work was given recently by Prof. Virtanen in lectures delivered to the Netherlands Agricultural Society at Wageningen and to the Chemical Society of Zurich.

Earlier experiments showed that certain legumes, for example, red clover, did not grow so well when the nitrogenous food derived from the root nodules was replaced entirely by ammonium nitrate, but that peas utilised both forms of nitrogen equally well, whilst white clover responded better to ammonium nitrate than to the food provided by the nodule bacteria. These and other observations led to the hypothesis that legumes take up their nitrogen from the nodules in the form of organic compounds, and that these compounds constitute the best form of nitrogennutrition for certain legumes. It was also found that these compounds diffuse into the surrounding soil, from which they are taken up by growing plants, like Gramineae. Both pot and field experiments have shown that, when the $p \mathrm{H}$ of the soil does not fall below 6 , one pea plant can provide sufficient nitrogen for two oat plants, but if the ratio of oats to peas is greater than two to one, both plants suffer from lack of nourishment.

Investigations were then made using sterilised sand and pea seeds inoculated with nodule bacteria, and it was found that the nitrogenous matter that diffused into the sand consisted entirely of organic compounds, which were almost equal in amount to the nitrogen compounds directly taken up from the nodules. It was also established that nitrogen compounds diffuse into the soil from young and fresh root nodules of the alder tree, thereby enabling the latter to assist the growth of other trees.

Analysis of the substances that diffused into sterile quartz sand from the roots of pea plants that had been strongly inoculated showed the chief constituent to be amino-acids : in percentages of total nitrogen, amino-nitrogen $77 \cdot 4$, ammonia-nitrogen 0 , amido-nitrogen $3 \cdot 30$, nitrogen in volatile bases $2 \cdot 73$, humin-nitrogen $2 \cdot 05$. 
The question then arose whether non-legumes could take up and utilise these nitrogen compounds directly, and to solve it pea and barley plants were grown together in sterile sand contained in three-necked Woulff bottles, the pea seeds being inoculated, and each kind of plant being made to grow out through a neck of the bottle. The nutrient solution used contained no nitrogen. The barley plants grew excellently, but in the control experiment, in which the pea seed was not inoculated, they failed to grow. It was also found that barley, as well as peas, grew exceedingly well right up to the flowering or fruiting stage when they were fed with an aqueous extract of a low moorland soil that contained no ammonia or nitric nitrogen; they also grew well when they were fed in sterile culture with peptone (Witte) as their sole source of nitrogen. It is therefore concluded that higher plants can take up and utilise directly organic nitrogen compounds present in soils before their nitrogen is mineralised by bacteria or other micro-organisms.

The nature of the organic nitrogenous compounds that are of value to plants, as well as their percentage utilisation, is now being investigated. Earlier experiments in which red and white clovers were grown under sterile conditions inside glass flasks showed that red clover responded well to hydrolysed casein and to aspartic acid, and less well to ammonium salts and nitrate, whereas with white clover the results were exactly opposite. In these experiments the hydrogen ion concentration of the nutrient solutions containing hydrolysed casein and aspartic acid remained constant, showing that the nutritional effects were due to the amino-acids themselves and not to ammonia that might be liberated by their decomposition. The later experiments, in which the plants were grown with their roots in sterilised nutrient solution or sand and their upper parts in the air, have shown that aspartic acid is a very good nutrient for legumes, but not for Gramineos, and that asparagin is excellent for barley, but much less good for Leguminosa.

The manner in which the plants utilise such organic compounds can at present only be conjectured. On one hand, it may be that enzymes like urease, asparaginase or aspartase liberate ammonia from these organic compounds when they are still in the root system, and that the plants utilise this ammonia to synthesise protein. On the other hand, it is also possible that the amino-acids may be utilised directly for the synthesis of protein.

\section{Enzymes: A Discovery and its Consequences}

By Dr. E. F. Armstrong, F.R.s.

$\mathrm{I}^{\mathrm{T}}$ is a hundred years since Payen and Persoz discovered diastase and recognised it as a ferment. To-day the ferments, or enzymes as we have preferred to call them, are in the forefront of interest as the factors concerned in all those chemical changes in the cell which in their totality are termed vital changes : they may indeed compose those invisible genes which make up the chromosomes. The discovery of diastase, apart from its broader consequences, has had a farreaching effect also in introducing science into one of the oldest industries, one already established on the Nile in the days of the Egyptians, that of brewing: the determination of diastatic power is to-day one of the first exercises which is performed on the new season's crop of barley and malt. The studies on diastase made in the cause of brewing have in turn enriched chemical science, and there is a notable list of eminent brewers' chemists to inscribe on the roll of honour of enzyme pioneers.

The discovery of Payen and Persoz in 1833 followed an observation by Kirchhoff that germinating barley grains contained a principle capable of converting starch into sugar, a power which Leuchs discovered was also possessed by human saliva. Payen and Persoz found out how to extract ground germinating barley with water and, after filtration, precipitate a white flocculent material by means of alcohol, which when dried and redissolved turned starch into sugar. Their procedure was that which is followed to-day in extracting an enzvme, though our hands have gained not a little cunning in concentrating the enzyme and freeing it from this and that impurity, largely as the result of methods devised and elaborated by Richard Willstatter, who was Davy medallist of the Royal Society in 1932. The active material was named 'diastase' by its discoverers. It was found by them to be present in other cereals during germination, and during the 'seventies, numerous workers established that diastase is very generally present in vegetable cells so long as the latter are living. In the meantime, Miahle had made a diastase by adding alcohol to saliva, and other observers found it in the animal pancreas. Animal diastase is generally referred to as ptyalin in the literature and in many textbooks the term amylase is preferred to diastase; a proper sense of historical loyalty should, however, make us adhere to the older term.

In any mention of the diastase story, the work of Horace Brown and Morris in connexion with the translocation of starch comes immediately to mind. The formation of diastase in foliage leaves during darkness and its conspicuous diminution during bright sunshine, were discovered by them and used to explain the behaviour of starch, which varies in amount in the opposite sense during the twenty-four hours. Classical also are the studies of Cornelius O'Sullivan at Burton-onTrent of the action of malt diastase on starch, published in 1876 : they mark a beginning of the efforts to establish the kinetics of enzyme reactions and also to unravel the structure of starch itself 